REMARKS

ON

\section{THE FUNCTIONS OF THE LIVER IN JAUNDICE.}

By J. WICKHAM LEGG, M.D.,

Fellow of the Royal College of Physicians, and Demonstrator of Morbid Anatomy in St. Bartholomew's Hospital.

Ir is well known that, in the old system of physiology preserved to us by Galen, the liver, besides being the source of the movement of the blood, was likewise the centre of animal heat and of sanguification. Shortly after the discovery of the circulation by Harvey, the liver was robbed of all its functions save that of bile-making. Strange to say, in our time, the liver has been restored to all the functions, nay, more than all, that it possessed in the belief of Galen, and physiologists are now well assured that the liver is the centre of sanguification, of animal heat, of bile-making, as well as of certain other functions not dreamt of by the ancients.

To those who watch the flux and reflux, the Euripus of medical opinion, this return to ancient beliefs (if I may so use the word) is most interesting. Astrology, the influence of the planets upon human destiny, would, if discussed by the encyclopadists, have been set down among the grossest superstitions of ancient and medixval writers; yet now modern science acknowledges the great influence which the planets have upon the sun-spots, and these again influence many terrestrial phenomena, and consequently mankind. Again : the chemists tell us of the belief now gaining ground that the elements are not separated by the hard and fast line formerly supposed, but that it is highly probable that all the elements have some common source; thus the theory of the philosopher's stone and the search after the transmutation of metals no Jonger appears to be the impossibility which fifty years ago it was believed to be.

To return to physiology. The most important function of the liver known to physiologists is its power of making glycogen, a discovery made not many years ago by Claude Bernard. Upon this function of the liver depend apparently sanguification, nutrition, and, indeed, animal heat ; for Claude Bernard has found that the blood issuing from the hepatic vein is the warmest of all in the body, and is invariably of a higher temperature than that in the portal vein or arterial system. Another function greatly dependent upon the glycogenic function is the making of bile. A fourth function, according to Meissner, is the secretion of the greater part of the urea excreted by the kidneys. The object of this paper is to inquire what becomes of these functions in jaundice, whether they continue unchanged or are greatly or slightly impaired. It has, I think, been hitherto too readily assumed by patho. logists, in their reasonings on the phenomena of jaundice, that the liver continues its functions just as in health.

First, as to the glycogenic function of the liver. Whether this were injured or not appeared to me so important a point to establish on a firm basis, that $I$ made a large number of observations upon animals in order to decide it. After ligaturing the bile-duct, I found that, whether the animal died within a few hours or a few days, in all cases alike glycogen was absent from the liver. The absence of the glycogen was constant. It is not, therefore, merely the outcome of the temporary disturbance of the operation, but a permanent change in the function of the liver. The glycogen, however long the animal lived, in no case returned.

These observations have been confirmed by von Wittich; so that it may now be taken as an established fact that, in complete obstruction to the bile-duct, the glycogenic function of the liver is abolished. In like manner, puncture of the fourth ventricle after ligature of the duct causes no sugar to be present in the urine.

To one acquainted with the rudiments of physiology, it will be unnecessary to point out the importance of these results. They suggest a reason for the wasting often seen in jaundice, and which often occurs rapidly, and for the sense of weakness and inability to exert themselves of which some patients complain.

Another function of the liver is the preparation of bile. The three important constituents of the bile are the bile-acids, the bile-pigments, and cholestearine. Of these, the bile-acids are the greatest both in amount and in physiological value. Taking seventeen grammes as the very lowest estimated amount of dry bile which a man daily excretes, twelve grammes of this will be formed by bile-acid salts, about three by the bile-pigment and mucus, and the remainder by the cholestearine and inorganic residue. If the functions of the liver continue uninjured in jaundice, the whole of this should be excreted by the kid. neys, as it is well known none other of the secretions but the urine contain either bile-pigment or bile-acids. It is true that the sweat may sometimes contain bile-pigment, but the amount lost in this farhion must be exceedingly small. What, howcver, is the real amount passed out of the body by the kidneys? If Schwanda's estimations may be trusted, the amount of bile-pigment passed by the urine in jaundice is very small. The highest figure which he gives is .015 $5 \mathrm{rm}$. in the twenty-four hours. It has been seen that the amount secreted in health and passed into the duodenum is at the lowest reckon. ing two gramines. The same of the bile-acids. The smallness of their amount in the urine for a long time caused their presence alto. gether to be overlooked; but all observers are now agreed that the bile-acids, though in small quantity, are really present in the urine. The amount is very small. No one has found more than 3 gramme of bile-acids excreted in the twenty-four hours : yet, if the functions of the liver continue uninjured, at least twelve srammis should pass out of the system. This difficulty has been felt by physiological chemists, and Ernst Bischoff has proposed a theory to escape from it. He supposes that the bile-acids are oxidised in the blood; not all of them, but that they are only oxidised when their amount reaches a certain figure; just as a certain amount of sugar may be injected into the blood, part of it passing out by the kidneys, the remainder being oxidised in the blood. This theory is one to which assent can hardly be given, as the bile-acids have already attained a high degree of oxidisation, and it is well known how well they resist oxidising changes, as putrefaction, and chemical agents, as sulphuric acid. It seems more reasonable to suppose that very little indeed of the bile-acids are secreted. It is a widespread belief among physiologists that the albuminous principles of the food are split up in the liver into glycogen and bile-acids ; the glycogen taking the carbon, hydrogen, and oxygen; the bile-acids the same elements combined with sulphur and nitrogen. Now, if the glycogenic function of the liver cease, what becomes more probable than that the bile-making function of the liver should cease or be greatly impaired? There is a well known example of the dependence of these two functions one upon another in the limax flail, first pointed out by Bernard. In this mollusc, the bile and glycogen are secreted alternately, one during digestion, the other after, and so on. So, on $a$ priori grounds, it is very likely that, when once the glycogenic function of the liver is lost, the bile would cease to be secreted as well. Lately, several observers have noticed that as jaundice progresses the bile-acids become less in quantity, and, as Golowin has found, even (lisappear from the urine altogether at the end of a long-continued jaundice.

The same reasoning may be applied to the bile-pigments; their source, however, is altogether different from that of the bile-acids, for it is probable that they are derived from the red corpuscles of the blood. There can be little doubt that the formation in the liver of bile-pigments must be decreased both from the small amount excreted by the kidneys and the slight staining of the tisstes even in severe jaundice compared with what would take place were the whole of the bile-pigments formed in health again passed into the blood. The colour of the patient would then be an Ethiopian blackness. Clinical observation also shows that the colour of jaundiced patients decreases as death draws near.

A third important function is, that the liver produces a large annount of heat. Bernard, some years ago, pointed out that the temperature of the upper part of the vena cava was higher than that of any other part of the body. This high temperature is, no doubt, due to the active chemical changes continually going on in the liver; and, if these changes cease, it must follow that the temperature itself wionld fall likewise. I have found, in some cases that I have exanised, the temperature in the upper part of the vena cava, forty-eight hours after ligature of the common duct, lowered to the temperature of the rectum. This lowering of the temperature is not, however, constant, and the investigation requires to be pushed farther than at present I have had the leisure to pursue it.

It has been long known that in jaundice the temperature of the body is below natural, provided the jaundice be not accompaniecl or cansed by any disease which of necessity begets fever. The lowering of the temperature in the hepatic veins, if confirmed, would serve (1) explain the low temperature of the whole body so commonly seen in jaindice ; for, if the liver be the great heat-giving centre of the body and it cease to give out heat, what sequence more natural than that the temperature of the whole body should be lowered?

Another revived Galenical function of the liver is its power of forming blood-corpuscles. It is well known that the blood of the hepatic 
vein contains a far greater number of both red and colourless corpuscles than that of the portal vein. The corpuscles of the portal vein show likewise under the microscope a more distorted shape than the blood-corpuscles of the general system; they are also richer in fat.

Now, in jaundice, if a number of old analyses may be trusted, the amount of blood-corpuscles is very greatly decreased. This has been explained by supposing that the bile-acids circulating in the blood destroy a large number of the red corpuscles. If, however, less than one gramme a day of bile-acids pass through the system, as I have endeavoured to show above may be the case, the bile-acids must be in so dilute a solution, that very little action upon the corpuscles can take place, in no way enough to account for the great diminution of the red corpuscles. If the liver be largely concerned, therefore, in the making of red blood-corpuscles, and its functions in jaundice be greatly impaired, it scems more probable that the anremic state of the blood in jaundice may be due to the injury done to the liver. The general state of nutrition in jaundice should, however, be kept in mind, as there can be no doubt that the removal of the bile from the' intestines causes a great disturbance in the phenomena of absorption.

The last function of the liver, the secretion of urea, rests upon a base by no means so well founded as the others. It is not a theory which, in the present state of knowledge, greatly commends itself to my mind, nor do the observations which I have made upon the urea in jaundice seem at all to confirm the theory. For, if most of the other functions of the liver known to us be greatly impaired or even entirely abolished, it would be strange if this alone should remain in unchanged vigour. And yet this is really the case; for, in a series of estimations which I have made, and published in a paper read in March last before the Royal Medical and Chirurgical Society, I did not find the urea so much diminished as on Meissner's theory might be looked for. The decreased amount could be readily accounted for either by the disease which caused the jaundice, the general health and food of the patient, rest in bed, and the like; while, in two cases in which the obstruction to the ducts was most complete, and in which no other disease save the obstruction was present, the amount of urea reached its highest point. These results do not dispose me to look with great favour on Meissner's theory.

\section{THE B A L H A M Y T E R Y.}

By W. F. WADE, F.R.C.P.,

Physician to the General Hospital, Birmingham; etc.

Tine verdict of the last jury has been eminently satisfactory to a large number of the public, consisting chiefly, if not entirely, of those persons who, without waiting to hear the evidence, had come to the same conclusion at which the jury arrived. For my own part, having read the evidence daily in the Times' reports, I had come to think that, on the whole, an open verdict would be the proper one. Having since reread the same reports, in my judgment there is not sufficient evidence to say :

I. That Mr. Bravo died from taking tartar emetic;

2. That he did not take it accidentally :

3. That he did not take it suicidally;

4. That he was murdered;

5. That antimony was not given to him for the purpose of nauseating, without any intention either then or subsequently to take his life.

Whilst against any hypothesis which may be set up to account for his death, arguménts more or less strong, but in no case trivial, may be adduced, there seems to me a very considerable preponderance of evidence in fayour of suicide.

I. Did Mr. Bravo take antimony in the form of tartar emetic?

Dr. Redwood, who made the analyses, is Professor of Chemistry to the Pharmaceutical Society; and, as his name does not appear in Churchill's Medical Directory as a medical man, it is fair to assume that he has no medical qualification. He distiuctly states that he did not detect tartar emetic, and that he only infers, as a matter of probability, that it was this drug, and not some other form of antimony, which had been taken. This inference was drawn partly from the symptoms; but when other preparations, such as James's powder or black sulphuret, produce symptoms at all, these are indistinguishable from those produced by tartar emetic. It is needless to say that any one might obtain any quantity of James's powder with little if any difficulty. With regard to black sulphuret, Pereira says (Materia Medica, vol. i, p. 397) it was known in the most ancient times, being used by the Asiatic and Greek ladies as a pigment for the eyebrows. Dr. A. T. Thomson says (vol. ii, p. 22 I, note), "It was with the sulphuret of antimony that the Greek and 'Turkish ladies, to use a Scriptural phrase, 'put their eyes in mourning'. The sulphuret was applied witinin the eyelids, and produced a peculiar softness of expression". Further, "prepared sulphuret of antimony is the sulphuret of commerce levigated with water on a porphyry stone. It is inodorous, insipid, of a dark leaden grey hue, and stains the fingers when handled. It is insoluble in water and in alcohol, partially soluble in the vegetable acids, and consequently in wine. As an emetic, it is uncertain in its effects. If the stomach be acescent, it operates with violence". It is superfluous to point out the acescent state of the sto. mach an hour after dinner, and the presence of Burgundy in the stomach, in this case. It con ains, on an average, about 70 per cent. of metallic antimony. It is curious to note that the vomit in this case had been so drenched with heavy rain that only the solid portions remained, and to note at the same time that tartar emetic is easily soluble, and had, in all probability, if taken at all, been taken in solution, and would, therefore, be easily washed away; whereas the black sulphuret is insoluble in water, and would, therefore, if mixed with solid matter, be unaffected by the rains. It is also a fact, that Mrs. Bravo had some means of darkening her hair. I may add, that if it were proved that Mr. Bravo took this drug and not tartar emetic, the suspicion of suicide would amount almost to a certainty. James's powder is also, in great part, if not entirely, insoluble in water.

From these considerations my opinion is drawn, not only that it is not certain that the antimony was taken in the form of tartar emetic, but that it is more probable that an insoluble preparation of antimony had been taken.

2. That he did not take it accidentally.

No antimony has been traced to the possession of any one in the house, excepting some homcopathic preparations which Dr. Redwood asserted to be so weak as to be innocuous. The former possession of tartar emetic by the coachman Griffiths, and some other circumstances, may be sufficient to excite a suspicion against certain parties, but are quite insufficient to preclude the possibility of accidental death.

3. That he did not take it suicidally.

This part of the subject divides itself primarily into two heads : $a$. Reasons for thinking that he did not commit suicide; and $b$. Reasons for thinking that he did.

His stepfather states that he was a very courageous man, and that he had gathered from him in general conversation that he considered suicide cowardly. Whether suicide is a cowardly or a courageous act, is a question upon which much might be said. But the opinions expressed by poor Mr. Bravo in cosy chat with his stepfather before his marriage, have little to do with his actions or views under very different circumstances. Besides, crimes are constantly committed by the very last person who would, in the opinion of his intimates, be likely to perpetrate them. Look at the frequent embezzlements by highly well. conducted persons. Several eminent alienists have publicly expressed the opinion, in which I entirely concur, that he was a man very likely, under suitable conditions, to commit an impulsive suicide. The evidence of educated and highly respectable friends, whose opinions on this sub. ject are those of unskilled witnesses, was allowed to go unchallenged
to the jury.

A second reason is that, had he desired to commit suicide, his medical knowledge would have prevented him from using tartar emetic. His stepfather stated that he took great interest in surgical matters, and attended operations at one of the hospitals occasionally; and again, "I say my son had a liking for surgery, but, as distinguished from surgery, his medical knowledge was only general". Some of his legal friends gave somewhat similar evidence.

His uncle by marriage, a London hospital surgeon, said that Mr. Bravo knew something of suryery; and, as medicine and surgery are now-adays intimately connected, he supposed he knew something of medicine. He also said : "He never went to any other part of the hospital but the operating-room; and admitted that he could not tell a man who was likely to commit suicide from one who was not."

In the country of the blind, the one-eyed man is king. It is quite possible that, by seeing and talking of a few operations, he might get the credit with his companions, who knew nothing, of having medical knowledge. There is no evidence worthy of the name that his skill in medicine would lead him to avoid antimony or select any other drug for suicidal purposes; besides, the presumption is that, if he committed suicide, he did it impulsively, and in such cases people are not particular as to the means. But, suppose he had acquired thera. peutical or toxicological knowledge of antimony as a poison, what would he have learnt? Firstly, that tartar emetic has scarcely, if ever, been 